Bull. Mater. Sci., Vol. 22, No. 3, May 1999, pp. 439-441. (C) Indian Academy of Sciences.

\title{
Second-harmonic generation in distributed feedback ferroelectric liquid crystal cells
}

\author{
H HOSHI*, D-H CHUNG, K ISHIKAWA and H TAKEZOE \\ Department of Organic and Polymeric Materials, Tokyo Institute of Technology, O-okayama, Meguro-ku, \\ Tokyo 152-8552, Japan
}

\begin{abstract}
The helicoidal structures in ferroelectric liquid crystals can be utilized to realize a special phase matching for the second-harmonic generation (SHG) when two counter-propagating fundamental waves are normally incident and the wavelength of the second-harmonic wave is near the selective reflection band edge. In this paper, we report the thickness and polarization dependences of the SHG thus observed. By comparing the experimental results with the theoretical ones developed, good agreement was confirmed.
\end{abstract}

Keywords. Second-harmonic generation; ferroelectric liquid crystal; helicoidal structure; phase matching; distributed feedback cavity.

\section{Introduction}

The helicoidal structures in liquid crystals (LCs) provide more possibilities to realize phase matching conditions than those in homogeneous material. Shelton and Shen $(1970,1971,1972)$ demonstrated many phase matching conditions for the third-harmonic generation (THG) using cholesteric LCs. Kajikawa et al (1992) and Furukawa et al (1995) studied the second-harmonic generation (SHG) in the smectic-C* $\left(\mathrm{Sm}-\mathrm{C}^{*}\right)$ phase of ferroelectric LCs, using a normally incident fundamental wave. They observed significant SHG, when the optical pitch was close to the wavelength of SHG. Theoretical studies by Belyakov and Shipov $(1981,1982)$ and Copic and Drevensek-Olenik (1996) suggested that a remarkable additional enhancement could be realized when the wavelength of the harmonic is close to the edge of the selective reflection band. In particular, they pointed out the possibility that a special type of phase matching for SHG is realized when two counter-propagating fundamental waves create SH waves near the selective reflection band edge. They also suggested that the enhanced SHG observed by Kajikawa et al (1992) and Furukawa et al (1995) originates from this mechanism via weak reflection from the back surface of the cell. Yoo et al (1997) used the geometry of two counter-propagating fundamental waves and confirmed the theoretical prediction by Copic and Drevensek-Olenik (1996). In this paper, the thickness and polarization dependences of this enhanced SHG were measured and compared with the theory.

\section{Experiment}

SHG was measured using normally incident two counterpropagating fundamental waves of $\mathrm{Nd}$ : YAG laser (wave-

*Author for correspondence length $1.064 \mu \mathrm{m})$. Figure 1 shows the optical geometry, where two quarter wave plates produce left $(l)$ - or right (r)-handed circularly polarized waves. The LC cell used was described in the preceding papers (Furukawa et al 1995; Yoo et al 1997). The LC molecules form a right-handed helicoidal structure whose axis is normal to the glass substrates. Three cells with thicknesses of $28.5,62 \cdot 0$, and $87.0 \mu \mathrm{m}$ were prepared.

\section{Results and discussion}

The optical pitch for this LC cell was reported previously. The optical pitch is $0.532 \mu \mathrm{m}$ at about $39-43^{\circ} \mathrm{C}$ (Furukawa et al 1995; Yoo et al 1997). The lower temperature results in shorter optical pitch. Since the optical pitch slightly depends on the cell because of the different anchoring conditions, we use $T_{\mathrm{C}}$ to denote the temperature where the optical pitch matches with $0.532 \mu \mathrm{m}$. For the fundamental wavelength of $1.064 \mu \mathrm{m}$, the special phase matching is expected around this temperature. Figure 2 shows the $\mathrm{SH}$ intensity as a function of temperature using two linearly polarized counter-propagating fundamental waves. Significant enhancement is actually observed when the selective reflection band is near the wavelength of SHG. The following features should be noted: (i) the SHG signal drastically increases with increasing cell thickness and (ii) the peak position shifts toward the lower temperature side with increasing cell thickness.

To understand these features we simulated SHG using the exact model by Drevensek-Olenik and Copic (1997). In this model, three (left, middle, right) layers were considered. The left and right layers are isotropic substrates whose dielectric constants are $\varepsilon_{\mathrm{s}}$ and $\varepsilon_{\mathrm{s}}^{\prime}$ at fundamental frequency $\omega$ and at $\mathrm{SH}$ frequency $2 \omega$, 
respectively. The middle layer (thickness $d$ ) consists of Sm-C* structure which has local $\mathrm{C}_{2}$ symmetry and forms helicoidal structure (pitch $p$ ) normal to the substrate surface. The local Cartesian coordinate systems $\left(e_{1}, e_{2}, e_{3}\right)$ was introduced, where $e_{2}$ and $e_{3}$ axes are parallel to the $\mathrm{C}_{2}$ axis and the major principal axis of the dielectric constant (nearly along the molecular long axis), respectively. In this coordinate system, the dielectric constants and the nonzero components of the nonlinear optical susceptibility are $\varepsilon_{1}, \varepsilon_{2}, \varepsilon_{3}$ at $\omega\left(\varepsilon_{1}^{\prime}, \varepsilon_{2}^{\prime}, \varepsilon_{3}^{\prime}\right.$ at $2 \omega)$ and $\chi_{123}, \chi_{112}, \chi_{332}, \chi_{222}$, where Kleinman's symmetry was assumed for $\chi^{(2)}$. The tilt angle $\theta$ is the angle between $e_{3}$ axis and the helicoidal axis.

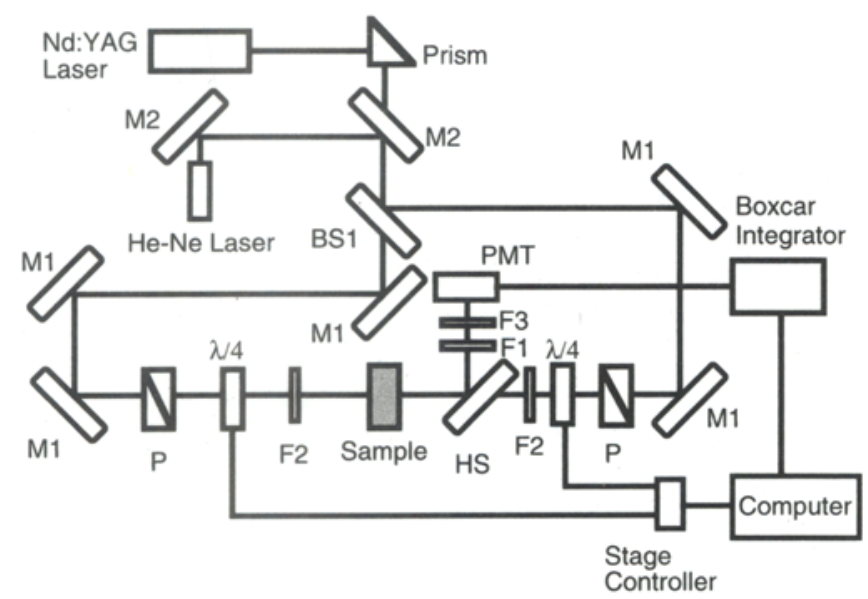

Figure 1. Optical geometry for SHG measurement.

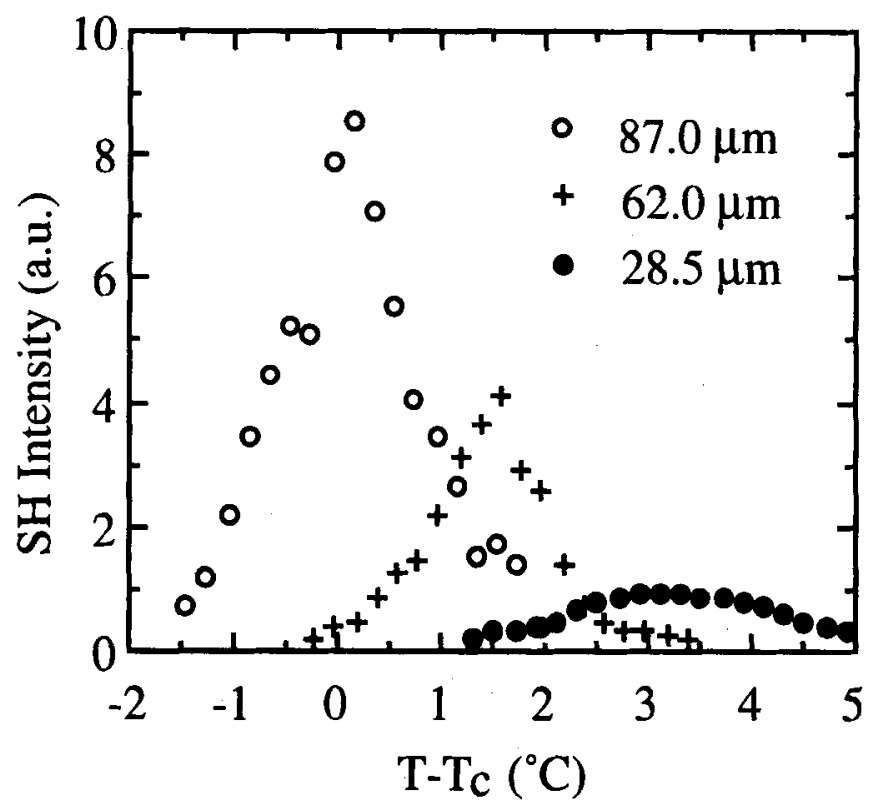

Figure 2. Experimental results on the temperature and thickness dependences of the SH intensity.
The following values were used for simulation; $\varepsilon_{\mathrm{s}}=\varepsilon_{\mathrm{s}}^{\prime}=2 \cdot 25, \varepsilon_{1}=\varepsilon_{1}^{\prime}=\varepsilon_{2}=\varepsilon_{2}^{\prime}=2 \cdot 2$ and $\varepsilon_{3}=\varepsilon_{3}^{\prime}=2 \cdot 8$, which were measured by determining the deflection angle of an $\mathrm{Ar}$ laser by a wedge cell in the $\mathrm{Sm}-\mathrm{A}$ phase. The tilt angle $\theta$ was $23^{\circ}$, which was determined under a microscope by applying an $\mathrm{AC}$ electric field to a homogeneously aligned cell. The following tentative values were used for $\chi^{(2)}$ components; $\chi_{123}=2$, $\chi_{112}=1, \chi_{332}=3, \chi_{222}=2$.

Figure 3 shows the calculated result for linearly polarized two counter-propagating waves. The edges of the selective reflection band are located at $\lambda_{2}$ and $\lambda_{13}$, which are expressed as

$$
\lambda_{2}=\sqrt{\varepsilon_{2}^{\prime}} p, \quad \lambda_{13}=\sqrt{\frac{\varepsilon_{1}^{\prime} \varepsilon_{3}^{\prime}}{\varepsilon_{1}^{\prime} \sin ^{2} \theta+\varepsilon_{3}^{\prime} \cos ^{2} \theta}} p
$$

For $p=0.35867 \mu \mathrm{m}, \lambda_{2}=0.5320 \mu \mathrm{m}$ and $\lambda_{13}=0.5409 \mu \mathrm{m}$, and for $p=0.35276 \mu \mathrm{m}, \lambda_{2}=0.5232 \mu \mathrm{m}$ and $\lambda_{13}=$ $0.5320 \mu \mathrm{m}$. Among these possibilities, the enhancement occurs only near $p=0.35867 \mu \mathrm{m}$, indicating that the enhancement occurs only at $\lambda_{2}$ edge of the selective reflection band. The simulation shows that the pitch $p_{\text {max }}$, which corresponds to the maximum SH intensity, converges to $p=0.35867 \mu \mathrm{m}$ with increasing cell thickness. Thus, the thickness-dependent peak shift is well described by the theory. According to the theory, the maximum SH intensity is proportional to $d^{4}$ as pointed out by Belyakov and Shipov (1981). For realizing $d^{4}$ dependence, it is necessary to prepare the cell with high quality and stability.

Figure 4 shows the temperature dependence of SHG for three polarization conditions, where the polarized

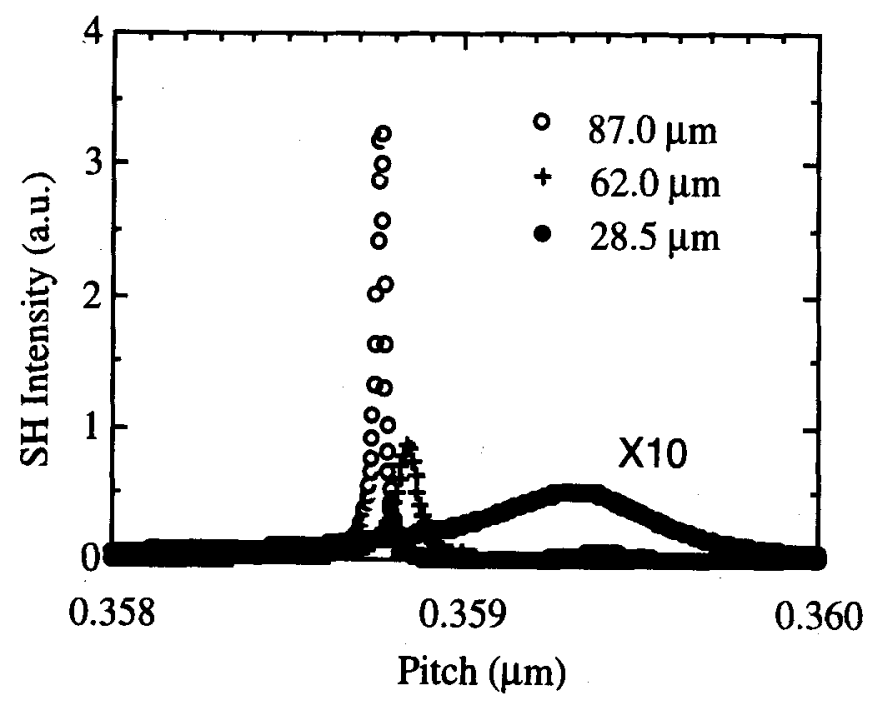

Figure 3. Calculated results of the pitch and thickness dependences of SHG. The SH intensity for a $28.5 \mu \mathrm{m}$ thick cell is magnified by 10 . 


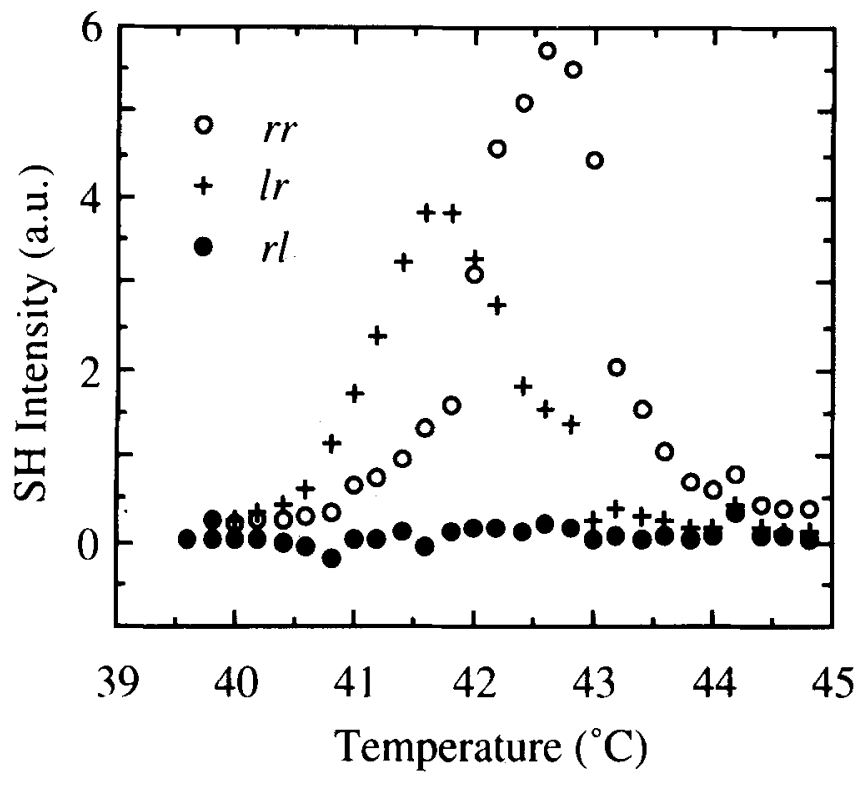

Figure 4. Experimental results of the temperature and polarization dependences of the SH intensity. Cell thickness was $87 \mu \mathrm{m}$.

states of the fundamental waves are specified in the order of the left and right incident waves. The following features should be noted: (i) enhanced SHG was observed for $r r$ polarization, (ii) enhanced SHG with lower peak intensity was observed for $l r$ polarization. The peak position under $l r$ condition was slightly shifted from $r r$ condition and (iii) no enhancement occurred for $r$ polarization.

The pitch and polarization dependence of SHG were calculated under the condition that two counterpropagating waves with equal amplitudes are incident from the left and right sides of the cell in the polarization states of $r r, l r$, and $r l$. The SHG intensity emitted toward the right side was calculated (figure 5). The experimental results shown in figure 4 qualitatively agree with the simulation. This comparision suggests that the peak for $r r$ condition will be sharper and larger. The variation of the temperature and the surface anchoring may cause the broadening of the peak and the reduction of the peak intensity.

\section{Conclusion}

The enhancement of SHG in FLC cells due to helicoidal distributed feedback cavity effect was studied. The

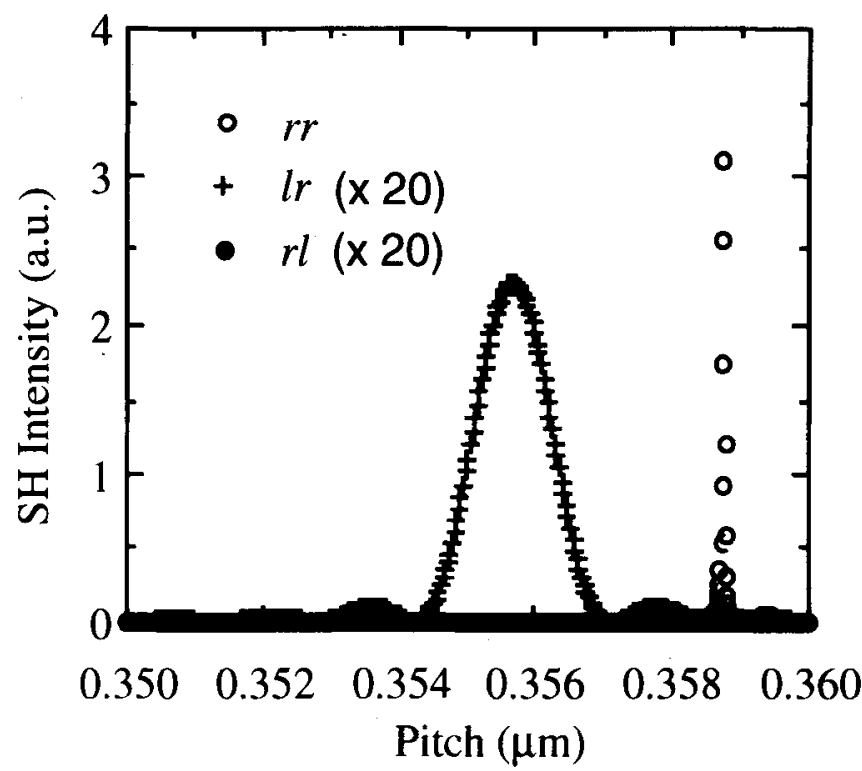

Figure 5. Calculated results of the polarization and pitch dependences of the SH intensity. The SH intensities for $l r$ and $r l$ polarizations are magnified by 20 .

experiment and simulation were made for the dependences on the thickness and incident polarization. The good agreement between the experiment and the theory based on the exact treatment by Drevensek-Olenik and Copic (1997) was confirmed.

\section{Acknowledgements}

The authors thank Dr M Schadt, ROLIC, for supplying LC material used.

\section{References}

Belyakov V A and Shipov N V 1981 Phys. Lett. A86 94 Belyakov V A and Shipov N V 1982 Sov. Phys. JETP 55674 Copic M and Drevensek-Olenik I 1996 Liq. Cryst. 21233 Drevensek-Olenik I and Copic M 1997 Phys. Rev. E56 581 Furukawa T, Yamada $\mathrm{T}$, Ishikawa $\mathrm{K}$, Takezoe $\mathbf{H}$ and Fukuda A 1995 Appl. Phys. B60 485

Kajikawa K, Isozaki T, Takezoe H and Fukuda A $1992 J p n J$. Appl. Phys. 31 L679

Shelton J W and Shen Y R 1970 Phys. Rev. Lett. 2523

Shelton J W and Shen Y R 1971 Phys. Rev. Lett. 26538

Shelton J W and Shen Y R 1972 Phys. Rev. A5 1867

Yoo J-G, Choi S-W, Hoshi H, Ishikawa $\mathrm{K}$, Takezoe $\mathbf{H}$ and Schadt M 1997 Jpn J. Appl. Phys. 36 L1168 